\title{
Le pas de valse à trois temps de l'enseignement de la proctologie : entre opportunisme, manque de considération et déception...*
}

\author{
L. Siproudhis - C. Brochard - V. de Parades $\cdot$ L. Abramowitz $\cdot$ F. Pigot - G. Bouguen \\ (C) Lavoisier SAS 2019
}

"Cette université avait le malheur de n'être fameuse que par sa théologie scolastique et par ses disputes "

Voltaire, Mœurs

L'enseignement de la proctologie traverse actuellement une période de turbulence pour ne pas dire de « maltraitance $\gg . .$. qui mérite que l'année 2019 lui apporte un peu d'apaisement et un certain regard confiant tourné vers l'avenir. La réforme de l'internat, le mépris du plus grand nombre des universitaires français et la querelle médicochirurgicale des Anciens et des Modernes n'y sont pas étrangers.

\section{Satisfecit}

" Ainsin, estant toutes choses subjectes à passer d'un changement en aultre, la raison, $y$ cherchant une reelle subsistance, se trouve deceue »

\section{Montaigne, Essais II}

« Billet de satisfaction donné par le maître à son élève », nous dit Littré. C'est aujourd'hui une réflexion en miroir de cette définition qui est proposée lorsque le maître reçoit (ou non) le billet de satisfaction de son élève.

La proctologie a structuré depuis plus de 20 ans une formation ouverte aux hépatogastroentérologues et aux chirur-

\section{Siproudhis $(\bowtie)$}

CHU de Rennes, hôpital Pontchaillou,

2, rue Henri-Le-Guilloux, F-35000 Rennes, France

e-mail : laurent.siproudhis@chu-rennes.fr

C. Brochard · V. de Parades - L. Abramowitz · F. Pigot

Conseil d'administration de la SNFCP

Domus Medica, 79, rue de Tocqueville, F-75017 Paris, France

L. Abramowitz

Membre du COPIL DES Île-de-France

G. Bouguen

Coordinateur DES HUGO

\footnotetext{
* Tous sont des enseignants actifs du DIU de proctologie.
}

giens. Cette formation est dispensée sur la base d'une plateforme de téléenseignement depuis 2004 pour sa partie théorique et sur un mode de compagnonnage auprès de maîtres de stage en région pour sa partie pratique. Cette formation est exigeante, notamment dans la dimension de la formation chirurgicale des actes proctologiques, et tout particulièrement pour les jeunes hépatogastroentérologues qui souhaitent s'y engager. Cet enseignement a été structuré autour d'un diplôme interuniversitaire national et unifié pour compenser les importantes carences universitaires de nombreux CHU. La formation théorique est aujourd'hui entrée dans une période de pleine maturité. Elle implique à la fois des enseignants et des formateurs chirurgiens et hépatogastroentérologues. Elle a fait l'objet récemment d'une mise à jour. Au cours des cinq dernières années, plus de 1000 apprenants ont suivi cette formation. Les répondeurs d'un sondage récent confortent les options prises d'une formation de deux ans : un premier niveau d'enseignement simple et polyvalent pour un exercice minimal de la proctologie et un second niveau de formation plus spécifique ouvrant sur un exercice chirurgical.

Quand elle est menée à son terme, cette formation permet un exercice de pratique proctologique dans $84 \%$ des cas et un exercice de la chirurgie proctologique dans $47 \%$ des cas. Satisfaction également parce que l'enseignement de la proctologie a ouvert la voie à un nouveau mode de transmission (e-learning) et parce qu'il a accueilli en d'autres temps, au sein de sa plateforme, d'autres formations naissantes de la discipline (qui s'en souvient? En cela, l'enseignement de la proctologie était un incubateur de formations). Satisfaction encore parce que le premier niveau de formation intègre aujourd'hui les bases d'enseignement DES en hépatogastroentérologie pour tous les internes DES, sans distinction entre les CHU qui offrent une formation proctologique et ceux (si nombreux) qui n'en offrent pas. Parmi les internes DES qui ont suivi cette formation, le volume d'enseignement est jugé suffisant et adapté à l'attente dans 62 et $79 \%$ des cas respectivement. Un tiers d'entre eux souhaiteraient poursuivre une spécialisation médicochirurgicale ou acquérir des compétences supplémentaires en proctologie après la formation offerte pendant le DES. Satisfaction finalement 
parce que l'ouverture de cette formation théorique permet à des médecins francophones étrangers d'accéder au parcours soit dans le cadre d'une formation diplômante (Belgique, Maghreb, Moyen-Orient), soit dans le cadre de l'accompagnement d'enseignement dans des pays qui en ont fortement besoin (Cambodge, Afrique subsaharienne). Qualitativement également, les signaux sont nombreux parmi les internes et les assistants qui demandent une formation spécifique auprès des centres de référence afin d'acquérir une compétence et les capacités à exercer une activité chirurgicale proctologique simple et de recours.

\section{Opportunisme, manque de considération et déception}

"Vous vous taisez, madame, et ce cruel mépris

N'a pas du moindre trouble agité vos esprits » Racine, Andromaque, IV, 2

La richesse de l'enseignement de la proctologie puise ses racines dans la pluridisciplinarité et le souci permanent d'une complémentarité d'enseignement offert à la fois aux hépatogastroentérologues et aux chirurgiens digestifs. Cette richesse et cette complémentarité sont au cœur même du projet d'enseignement. Elles sont toutes les deux le ciment de la Société nationale française de coloproctologie.

L'année 2016 et les projets de réforme du DES nourrissaient la perspective d'une formation spécialisée transversale (FST, ex-DESC) qui pouvait offrir un cadre de formation d'expertise à un petit nombre $(n=10$ par an) d'internes motivés (une année de formation dans un centre expert). Ce projet permettait d'accroître la lisibilité des centres de formation, de structurer la formation universitaire là où elle manquait, de faciliter les échanges entre les universités autour de postes d'internes budgétés et ciblés, de donner une vraie dimension universitaire à ce « parent pauvre » de la chirurgie digestive et de l'hépatogastroentérologie. Ce rendez-vous a été manqué.

La proctologie a peut-être servi de faire-valoir d'un certain volume de formation lorsque la maquette du DES d'hépatogastroentérologie était en quête d'une cinquième année. La proctologie a servi de chantage et de monnaie d'échange dans le différend qui oppose aujourd'hui les chirurgiens digestifs et les endoscopistes. La suppression des options et notamment de l'option de proctologie est intervenue dans le sillage immédiat de l'obtention de la cinquième année (Tableau 1). Il n'y a pas eu les discussions et les concertations souhaitées. La Société nationale française de coloproctologie s'est émue. De nombreux universitaires engagés dans la formation des hépatogastroentérologues et des chirurgiens digestifs n'ont pas compris les conséquences de ces choix. Ils n'ont pas compris parce qu'ils ne connaissent pas les contraintes et les difficultés de formation notamment chirurgicale (volume d'activité, gestuelle, indications, gestion des suites et des complications). Ils n'ont pas compris parce que, hépatologues, motriciens, endoscopistes ou oncologues, cette formation ne les concerne pas directement. Ils ne veulent pas non plus comprendre parce que discuter du maintien de cette filière de formation, c'est également évoquer les liens et les ponts à établir entre le monde chirurgical et celui des hépatogastroentérologues (aujourd'hui rendus étanches autour d'une endoscopie thérapeutique stigmatisée). Ils n'ont pas compris parce qu'ils sont à la recherche d'un cursus de formation homogène qui gomme les différences au sein du DES, mais qui efface également la diversité et le particularisme de cette grande discipline. Devant tant de désengagements, face à l'absence de discussion et de concertation, face à l'absentéisme des chirurgiens digestifs dans un tel débat, l'avenir de la formation questionne et invite à une réflexion commune. On est au cœur de la scolastique et d'une certaine forme de querelle des Anciens et des Modernes où s'agitent en toile de fond l'exercice de l'oncologie de spécialité et la chirurgie endoscopique. Ce dogmatisme stérilise et empêche le déploiement d'initiatives.

\section{La proctologie a besoin de vous}

\section{" Je me procurerai tous mes besoins, et, pourvu que je les aie, je ne me soucierai point que les autres soient misérables »}

\section{Montesquieu, Lettres persanes}

La vision jacobine d'un organe de décision centralisé conseille aujourd'hui de nous contenter de ce qu'on a construit et de ne pas faire de vague. Tout ne va pour autant pas pour le mieux, et c'est bien en sollicitant encore et toujours les bonnes volontés du groupuscule des proctologues lassés que le projet pourrait survivre. Le besoin le plus urgent concerne les stages pratiques et l'identification des maîtres de stage. Parmi les internes DES qui ont répondu à l'enquête de sondage, les trois quarts estiment qu'il est difficile de trouver un stage pratique au sein de son CHU ou en proximité. La problématique est similaire, à un moindre degré, lorsque les apprenants sont inscrits dans un parcours du diplôme interuniversitaire (42\%). Comment envisager la formation d'un futur gastroentérologue sans qu'il puisse disposer d'une formation pratique minimale ? Pour y répondre, nous avons besoin de vous. Vous qui êtes des proctologues avec un exercice épanoui et qui vous retranchez derrière l'argument d'un exercice libéral (qui vous a formés ? Qui vous a transmis sa connaissance pratique ? Comment avezvous été accompagnés ? N'est-il pas possible d'établir facilement des conventions de stage pratique pour un accueil, une journée par semaine, d'un interne DES dans une 
Tableau 1 L'historique d'un projet d'enseignement avorté

$08 / 02 / 2016$

$05 / 08 / 2016$

$07 / 12 / 2016$

$27 / 04 / 2017$

$27 / 06 / 2017$

$14 / 09 / 2017$

$26 / 10 / 2017$

$18 / 03 / 2018$

$22 / 03 / 2018$

$23 / 03 / 2018$

$09 / 04 / 2018$

$01 / 10 / 2018$

$11 / 10 / 2018$

$12 / 10 / 2018$

$30 / 10 / 2018$
Envoi de la cinquième version travaillée de la FST de proctologie médicochirurgicale qui reçoit votre plein soutien en intégrant la maquette d'enseignement du DES

Discussions délicates où il apparaît que cette FST est un outil de contrepartie à une FST d'endoscopie médicochirurgicale. La maquette de la FST est jugée mature et le dossier solide

Décision de suppression de la FST de proctologie au profit de la création d'une option. C'est une sortie de scène prudente qui fragilise le projet défendu dans sa double composante

Parution au Journal officiel de la maquette et de l'option de proctologie

La maquette de l'option est retravaillée, les objectifs pédagogiques et le volume de formation viennent grossir le dossier des justifications de la maquette à 5 ans

Confirmation du maintien de l'option de proctologie dans la maquette avec satisfecit

Dans le sillage de la réunion IGAS, les statistiques de formations des DU viennent nourrir le même objectif que celui de juin 2017. La proctologie est présente et apporte un volume certain à l'édifice Injonction d'organisation des options et des FST

Recensement national des capacités de formation pour les options et pour les FST par faculté Édition des référentiels de compétence en proctologie

Nouvelle sollicitation urgente pour connaître sur cinq ans le nombre d'apprenants inscrits en formation du DIU de proctologie ( $n=1004)$

Proposition et réalisation d'études de sondage de satisfaction et d'évaluation des besoins de formation au sein du DES et du DIU

Annonce de la maquette à 5 ans

Demande de structuration des objectifs de formation comprenant également les filières options

Annonce de la suppression des options sans aucune concertation ni réversibilité

FST : formation spécialisée transversale ; DES : diplôme d'études spécialisées ; DU : diplôme universitaire ; DIU : diplôme interuniversitaire ; IGAS : Inspection générale des affaires sociales

structure de type libéral ?). Vous qui êtes des universitaires et qui n'avez pas toujours la possibilité d'offrir tous les besoins de formation des internes DES que vous accueillez dans vos services : que faire quand cela concerne une activité de soins si courante que la proctologie ? Vous, universitaires de chirurgie, qui avez entamé une « croisade de libération » de l'endoscopie digestive alors même que la chirurgie proctologique est faite, en fin de programme opé- ratoire, par des CCA déroutés par les modalités de prise en charge et sans personne de recours.

Ressaisissons-nous pour cette spécialité si diverse qu'est l'hépatogastroentérologie. Ressaisissons-nous pour les futurs coloproctologues médicochirurgicaux de l'Hexagone.

Liens d'intérêts : les auteurs déclarent ne pas avoir de liens d'intérêts. 